\title{
RICCI and Matter Collineations of SOM-ROY Chaudhary Symmetric Space Time
}

\author{
MUHAMMAD RAMZAN*†, YAQOOB AHMED*, AND MUHAMMADRAFIQ MUFTI** \\ RECEIVED ON 07.02.2017 ACCEPTED ON 29.05.2017
}

[DOI: org/10.22581/muet1982/1802.07]

\begin{abstract}
This paper is devoted to explore the RICCI and MCs (Matter Collineations of the Som-Ray Chaudhary spacetime. The spacetime under consideration is one of the spatially homogeneous and rotating spacetimes. Collineations are the some kinds of the Lie symmetries. To discuss the required collineations we have used the RICCI and energy momentum tensors. As the RICCI tensor is formulated from the metric tensor, it must possess its symmetries. RCs (RICCI Collineations) leads to conservation laws. On the other hand for the distribution of matter in the spacetimes, the symmetries of energy momentum tensor or MCs provides conservation laws on matter field. Throughout this paper, these collineations are discussed by vanishing Lie derivative of RICCI and energy momentum tensors respectively. Complete solution of the RCs and MCs equations, which are formed in the result of vanishing Lie derivative are explored. Studying all these collineations in the said spacetime, it has been shown that RCs of the spacetime form an infinite dimensional vector space where as MCs are Killing vector fields.
\end{abstract}

Key Words: RICCI Collineations, Matter Collineations, Killing Vector Fields, Infinite Dimensional Vector Space.

\section{INTRODUCTION}

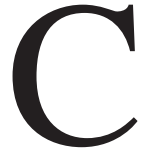

ollineations are the geometric assumptions which concern the "symmetries" of the metric defined by the Lie symmetries of either the metric itself or the tensors defined from the metric [1,2].

Theory of general relativity is basically the theory of gravitation and is nonlinear due to curved spacetimes. Einstein expressed this phenomenon in terms of set of gravitational field equations known as EFEs (Einstein Field Equations) are:

$$
R_{a b}-\frac{1}{2} R_{a b}+\Lambda g_{a b}+\kappa T_{a b}
$$

where $\mathrm{a}, \mathrm{b},=0,1,2,3$.

The left hand side of EFEs describes the geometry while the RHS is totally related to physics. In these equations, $\Lambda$ is called cosmological constant and $\kappa=8 \pi \mathrm{G} / \mathrm{C}^{4}$ is called coupling constant [3]. Due to high non linearity, it is very difficult to find the exact solutions of the system. To overcome this difficulty we introduce symmetry

†Corresponding Author (E-Mail: muhammad.ramzan@iub.edu.pk)

* Departmentof Mathematics, The Islamia University of Bahawalpur, Bahawalpur.

** Department of Computer Science,COMSATS Institute of Information Technology, Vehari, Pakistan.

This is an open access article published by Mehran University Research Journal of Engineering and Technology, Jamshoro under the CC by 4.0 International License. 
restrictions. These symmetry restrictions for mechanical problems reduce the degrees of freedom. Reduction of degrees of freedom simplifies the problem. The important symmetries which are frequently used in general relativity are KVFs (Killing Vector Fields), HVFs (Homothetic Vector Fields), CFVs (Conformal Vector Fields), CCs (Curvature Collineations), RCs and MCs [4]. Therefore, it is necessary to discuss the symmetries of different kinds in general relativity. Most of the symmetries give the conservation laws. Owing to that in this paper we have discussed two types of the important symmetries, namely RCs and MCs of said spacetime. It is obvious from the EFEs that RICCI and matter collineations are necessary to find the exact solutions of EFEs. MCs are totally related to the physical properties of the spacetimes, whereas RCs are concerned with the geometry of spacetimes $[2,5]$.There are many approaches to discuss RCs and MCs but the technique used to find RCs and MCs is vanishing Lie derivative of RICCI and energy momentum tensors along with the specified vector fields [6]. In this work, the system of nonlinear coupled partial differential equations generated by taking Lie derivative is solved by direct integration technique [5,7]. Normally in general relativity, Mis used for the manifold, which is four dimensional, connected, Hausdorffspacetime. The metric $\mathrm{g}$ hich contains all of the information about the geometry of the manifold is taken as Lorentzian with signature $(-,+,+,+)$. The RICCI and energy momentum tensors in component form which are connected with metric tensor $\mathrm{g}_{\mathrm{ab}}$, are denoted by $\mathrm{R}_{\mathrm{ab}}$ and $\mathrm{T}_{\mathrm{ab}}$ respectively. Comma, semicolon and $\mathrm{L}$ are the symbols used for partial, covariant and Lie derivatives respectively [5].

\section{RESULTS FOR RICCI COLLINEATIONS}

Consider a Som-Ray Chaudhary spacetime in the curvilinear coordinates $(\mathrm{t}, \mathrm{r}, \theta, \mathrm{z})$ labeled by $\left(\mathrm{x}^{0}, \mathrm{x}^{1}, \mathrm{x}^{2}, \mathrm{x}^{3}\right)$ respectively. Here, superscripts are not the powers. The line element is [8]:

$d s^{2}=-d t^{2}+d r^{2}+r^{2}\left(1-r^{2}\right) d \theta^{2}+d z^{2}+2 r^{2} d t d \theta$
The RICCI tensor and RICCI scalar in the usual notations are defined as $R_{a b}=R_{a c b}^{c}, R=g^{a b} R_{a b}$. The non-zero components of RICCI tensor and the value of RICCI scalar are given by $\mathrm{R}_{00}=2=\mathrm{R}_{11}, \mathrm{R}_{02}=\mathrm{R}_{20}=2 \mathrm{r}^{2}, \mathrm{R}_{22}=2 \mathrm{r}^{4}+2 \mathrm{r}^{2}, \mathrm{R}$ $=2$. The vector field $\chi$ is said to be RCs if it satisfies the relation $[6]$.

$\mathrm{L} \chi \mathrm{R}_{\mathrm{ab}}=0$

Equivalently,

$$
R_{a b, c} \chi^{c}+R_{b c} \chi_{a}^{c}+R_{a c} \chi_{b}^{c}=0
$$

where $\mathrm{a}, \mathrm{b}, \mathrm{c}=0,1,2,3$.

The explicit form of the Equation (2) can be written as a set of nonlinear coupled partial differential equations as:

$$
\begin{aligned}
& \chi_{, 0}^{0}-r^{2} \chi_{, 0}^{2}=0 \\
& \chi_{, 1}^{0}+\chi_{, 0}^{1}-r^{2} \chi_{, 1}^{2}=0 \\
& 2 r \chi^{1}-\chi_{, 2}^{0}+r^{2} \chi_{, 0}^{0}-\left(r^{4}+r^{2}\right) \chi_{, 0}^{2}+r^{2} \chi_{, 2}^{2}=0 \\
& \chi_{, 2}^{0}-r^{2} \chi_{, 2}^{2}=0 \\
& \chi_{, 1}^{1}=0 \\
& r^{2} \chi_{, 1}^{0}-\chi_{, 2}^{1}-\left(r^{4}+r^{2}\right) \chi_{, 1}^{2}=0 \\
& \chi_{, 3}^{1}=0 \\
& \left(2 r^{2}+1\right) \chi^{1}-r \chi_{, 2}^{0}+\left(r^{3}+r\right) \chi_{, 2}^{2}=0 \\
& \chi_{, 3}^{0}-\left(r^{2}+1\right) \chi_{, 3}^{2}=0
\end{aligned}
$$

From Equations $(3,4,7,9)$, we get

$$
\left.\begin{array}{c}
\chi^{0}=-\frac{1}{2} r A_{t}^{2}(t, \theta)+\int r^{2} A_{t}^{3}(r, \theta, z) d r+A^{5}(\theta, z) \\
\chi^{1}=A^{2}(t, \theta) \\
\chi^{2}=-\frac{1}{2} A_{t}^{2}(t, \theta)+A^{3}(r, \theta, z)
\end{array}\right\}
$$

Where $A^{2}(t, \theta), A^{3}(r, \theta, z)$ and $A^{4}(\theta, z)$ are functions of integration. To avoid from the lengthy calculations we 
only present the results. So, if one proceeds further, using the information obtained from Equation (12) in the remaining equations, we have the result.

$$
\left.\begin{array}{l}
\chi^{0}=r\left(c_{5} \cos \theta+c_{6} \sin \theta\right)+c_{8} \\
\chi^{1}=-c_{5} \sin \theta+c_{6} \cos \theta \\
\chi^{2}=-\frac{1}{r}\left(c_{5} \cos \theta+c_{6} \sin \theta\right)+c_{4}
\end{array}\right\}
$$

Finally, the RCs become

$$
\left.\begin{array}{l}
\chi^{0}=r\left(c_{5} \cos \theta+c_{6} \sin \theta\right)+c_{8} \\
\chi^{1}=-c_{5} \sin \theta+c_{6} \cos \theta \\
\chi^{2}=-\frac{1}{r}\left(c_{5} \cos \theta+c_{6} \sin \theta\right)+c_{4} \\
\chi^{3}=\xi\left(x^{a}\right), a=0,1,2,3
\end{array}\right\}
$$

Here $\xi\left(\mathrm{x}^{\mathrm{a}}\right)$ is an arbitrary function and $\mathrm{C}_{4}, \mathrm{C}_{5}, \mathrm{C}_{6}, \mathrm{C}_{8}$ are arbitrary constants. RCs after subtracting the Killing vector fields are:

$\chi=\left(0,0,0, \xi\left(x^{\mathrm{a}}\right)\right), \mathrm{a}=0,1,2,3$

Clearly RCs due to existence of the arbitrary function form an infinite dimensional vector space.

\section{MAIN RESULTS FOR MATTER COLLINEATIONS}

The expression of energy momentum tensor by considering the cosmological constant equals zero and the coupling constant equal to unity, takes the form [7]:

$$
T_{a b}=R_{a b}-\frac{1}{2} R g_{a b}
$$

The non-zero components of energy momentum tensor are:

$\mathrm{T}_{00}=3, \mathrm{~T}_{11}=1, \mathrm{~T}_{02}=\mathrm{T}_{20}=-3 \mathrm{r}^{2}, \mathrm{~T}_{22}=3 \mathrm{r}^{4}+\mathrm{r}^{2}, \mathrm{~T}_{33}=-1(17)$
The vector field $\eta$ is said to be MCs if the Lie derivative along this vector field vanishes. Mathematically it is represented by the relation.

$$
\underset{\eta}{L} T_{a b}=0
$$

Expansion of the Equation (18) is given by:

$$
T_{a b, c} \eta^{c}+T_{b c} \eta_{, a}^{c}+T_{a c} \eta_{, b}^{c}=0
$$

Here, a, b, c $=0,1,2,3$ and comma shows the partial derivative. The explicit form of the above Equation (18) with the help of Equation (17) can be written as set of ten nonlinear coupled partial differential equations:

$\eta_{, 0}^{0}-r^{2} \eta_{, 0}^{2}=0$

$3 \eta_{, 1}^{0}+\eta_{, 0}^{1}-3 r^{2} \eta_{, 1}^{2}=0$

$6 r \eta^{1}-3 \eta_{, 2}^{0}+3 r^{2} \eta_{, 0}^{0}-\left(3 r^{4}+r^{2}\right) \eta_{, 0}^{2}+3 r^{2} \eta_{, 2}^{2}=0$

$3 \eta_{, 0}^{3}-3 \eta_{, 3}^{0}+3 r^{2} \eta_{, 3}^{2}=0$

$\eta_{, 1}^{1}=0$

$3 r^{2} \eta_{, 1}^{0}-\eta_{, 2}^{1}-\left(3 r^{4}+r^{2}\right) \eta_{, 1}^{2}=0$

$3 \eta_{, 3}^{1}-\eta_{, 1}^{3}=0$

$(6 r+r) \eta^{1}-3 r^{2} \eta_{, 2}^{0}+\left(3 r^{4}+r^{2}\right) \eta_{, 2}^{2}=0$

$3 \eta_{, 0}^{3}+3 r^{2} \eta_{, 3}^{0}-\left(3 r^{4}+r^{2}\right) \eta_{, 3}^{2}=0$

$\eta_{, 3}^{3}=0$

From Equations $(19,20,23)$ and Equation $(28)$, we get: 


$$
\begin{aligned}
& \eta^{0}=-\frac{1}{6} z r A_{t}^{3}(t, \theta)+\int r A^{9}(t, \theta) d t+\int r^{2} A_{r}^{7}(r, \theta, z) d r \\
& -\frac{r}{3} A^{10}(\theta)+A^{12}(\theta, z) \\
& \eta^{1}=z A^{3}(t, \theta)-6 \int\left\{A^{9}(t, \theta) d t\right\} d t+t A^{10}(\theta)+A^{11}(\theta) \\
& \eta^{2}=-\frac{1}{6 r} z A_{t}^{3}(t, \theta)+\int \frac{1}{r} A^{9}(t, \theta) d t+A^{7}(r, \theta, z) \\
& \eta^{3}=r A^{3}(t, \theta)+A^{4}(t, \theta)
\end{aligned}
$$

Here all A's are the functions of integration to be determined with the help of direct integration technique. Again to avoid from tedious and lengthy calculation, only results are presented [6].Therefore, the solution of the system of equations from Equations (19-28) is given:

$$
\left.\begin{array}{l}
\eta^{0}=r\left(c_{11} \cos \theta+c_{12} \sin \theta\right)+c_{14} \\
\eta^{1}=-c_{11} \sin \theta+c_{12} \cos \theta \\
\eta^{2}=-\frac{1}{r}\left(c_{11} \cos \theta+c_{12} \sin \theta\right)+c_{10} \\
\eta^{3}=c_{6}
\end{array}\right\}
$$

Here all C,s are the arbitrary constants. Clearly, this system satisfies the Killing equation. Therefore, MCs of the given spacetime are the Killing vector fields.

\section{CONCLUSIONS}

From the above discussion we come to the following conclusions:

(i) When the RCs of the above spacetime has been discussed, it is found that RCs form an infinite dimensional vector space as mentioned in Equation (15).

(ii) In case of studying MCs of the above spacetime, it has been found that MCs are precisely the Killing vector fields as shown in Equation (30).

\section{ACKNOWLEDGEMENT}

The authors wish to express their gratitude to Prof. Dr. Ghulam Shabbir, Dean, Graduate Studies, Ghulam Ishaq Khan Institute of Engineering, Sciences \& Technology, Topi, KPK, Pakistan, for suggesting the problem and valuable discussion.

\section{REFERENCES}

[1] Hall, G.S., Roy, I., and Vaz, L.R, "RICCI and Matter Collineations of Spacetimes", General Relaitivity and Gravitation,Volume 28, No. 3, pp. 299-310, Germany, 1996.

[2] Sharif, M., and Sehar, A.,"Classifications of Spherically Symmetric Static SpacetimesAccording to their Matter Collineations", General Relativity and Gravitation, Volume 35, pp. 1093-1106, Germany, 2003.

Stephani, H., Kramer, D., MacCallum, M., Hoenselears, C., and Herlt, E.,"Exact SolutionsofEinstein's Field Equations", Cambridge University Press, UK, USA, 2009.

[4] Hall, G.S., "Symmetries and Curvature Structure in General Relativity”, World Scientific, Singapore, 2004.

[5] Saifullah, K., "RICCI Collineations of Cylindrically Symmetric Static Spacetimes", Ph.D. Thesis,The Quaide-Azam University, Islamabad, Pakistan, 2003.

[6] Katzin, G.H., Levine, J., and Davis, W.R, J., "Curvature Collineations: A Fundamental Symmetry Property of Space Time of General Relativity Defined by the Vanishing Lie Derivative of the Riemann Curvature Tensor", Journal of Mathematical Physics, Volume 10, pp. 617-629, USA ,1969.

[7] Sarwar, A.,"Matter Collineations in Som-Roy Chaudhary Spacetime", M.Phil. Thesis, The Islamia University of Bahawalpur, Pakistan, 2016.

[8] Krori, K.D., Borgohain, P., Kar, P.K., and Kar, D.D., "Exact Scalar and Spinor Solutions in Some Rotating Universes", Journal of Mathematical Physics, Volume 29, pp. 1645-1649, USA,1988. 